\title{
A NOTE ON THE ERGODIC THEOREMS
}

\section{YAEL NAIM DOWKER}

Introduction, definitions and remarks. The purpose of this note is to give an example of a measurable transformation of a measure space onto itself for which the individual ergodic theorem holds while the mean ergodic theorem does not hold.

Let $S$ be a measure space of finite measure, $m$ the measure defined on the measurable subsets of $S$, and $T$ a 1-1 point transformation of $S$ onto itself which is measurable (both $T$ and $T^{-1}$ transform measurable sets into measurable sets). Let the points of $S$ be denoted by $y$ and let $f(y)$ be any real valued function defined on $S$. We denote by $F_{h}(y)$ the average $(1 / h) \sum_{i=0}^{h-1} f\left(T^{i} y\right)$.

We shall say that the individual ergodic theorem holds for $f(y)$ if the sequence of averages $\left\{F_{h}(y)\right\}$ converges to a finite limit almost everywhere. If the individual ergodic theorem holds for every integrable function $f \in L_{1}(m)$ we shall say that the individual ergodic theorem holds (with respect to $m$ ). ${ }^{1}$

We shall say that the mean ergodic theorem holds in $L_{p}(m)(p \geqq 1)$ for a function $f \in L_{p}(m)$ if $F_{h}(y) \in L_{p}(m)$ for $h=1,2, \cdots$ and the sequence $\left\{F_{h}(y)\right\}$ converges in the norm of $L_{p}(m)$. If the mean ergodic theorem holds in $L_{p}(m)$ for every function $f(y) \in L_{p}(m)$ then we shall say that the mean ergodic theorem holds in $L_{p}(m)$.

The following relations between the two ergodic theorems are known: If $T$ is measure preserving, both the individual [1] and the mean $[4]^{2}$ ergodic theorems hold. Without assuming that $T$ is measure preserving, the mean ergodic theorem in $L_{p}(m)$ for any $p \geqq 1$ implies the individual ergodic theorem for all functions in $L_{p}(m)$ ( $[2$, p. 1061 ], see also $[3$, p. 539] for the case $p=1$ ).

The question arises whether, conversely, the individual ergodic theorem implies the mean ergodic theorem in $b_{p}(m)$ for some $p \geqq 1$. This question has significance only when $L_{p}(m)$ is transformed into itself by the transformation induced on it by $T$. For in this case and only in this case is it true that for any $f \in L_{p}(m)$ the averages $\left\{F_{h}\right\}$ also belong to $L_{p}(m)$ for $h=1,2,3, \cdots .^{3}$ We answer this question in

Received by the editors April 9, 1948.

1 The words in the parenthesis will be omitted if there is no reason for ambiguity.

${ }^{2}$ Only the case $p=2$ is proved in [4]; see [2, p. 1053] for all $p \geqq 1$. The numbers in brackets refer to the bibliography at the end of the paper.

${ }^{3}$ It is easy to give examples for which the individual ergodic theorem does hold while $L_{p}(m)$ is not transformed into itself. Such an example for instance is given if $T$ is periodic while $m$ is non-atomic and $T^{-1}$ is singular. 
the negative by constructing for each given $p \geqq 1$ an example of a transformation of a measure space $(S, m)$ onto itself for which (1) the individual ergodic theorem holds, (2) the mean ergodic theorem does not hold in $L_{p}(m)$, and (3) every function in $L_{p}(m)$ is transformed into a function belonging to $L_{p}(m)$.

Remark. Even though the individual ergodic theorem does not imply the mean ergodic theorem with respect to the original measure $m$ it is known [2, p. 1059] that in case the individual ergodic holds, it is possible to introduce a new measure $\mu$ defined on the measurable sets of $S$ such that $\mu$ has no more null sets than $m$ and $\mu$ is also invariant under $T$. It follows then from the statements made above that the mean ergodic theorem holds in $L_{p}(\mu)$ for every $p \geqq 1$.

The example for $p=1$. Let $S$ be the totality of all points on the circumferences $c_{1}, c_{2}, \cdots$ of a sequence of circles. Let the length of $c_{n}$ be $1 / 2^{n}$. Let the measure $m$ and the family of measurable sets in $S$ be the obvious ones determined by the Lebesgue measure on each of the circumferences. On each circumference $c_{n}$ we fix the polar coordinates $\rho=\left(1 / 2^{n} \cdot 2 \pi\right) \cdot e^{i \theta}$. Let $x=\theta / 2 \pi$. Let us divide $c_{n}$ into $2 n+2$ arcs, the end points of the arcs being $x=0, x=1 / 2$, and $x= \pm 1 / 2^{k+1}, k=1,2, \cdots, n$. The arcs are

$$
\begin{aligned}
A_{n k}: \frac{1}{2^{k}} & \geqq x \geqq \frac{1}{2^{k+1}}, \\
A_{n, n+1}: \frac{1}{2^{k+1}} & \geqq x \geqq 0,
\end{aligned}
$$

while if $n+2 \leqq k \leqq 2 n+2, A_{n k}$ is the reflection in $\theta=0$ of $A_{n, 2 n+3-k}$.

We define $T$ as follows: For the points of $A_{n k}$ let $T$ be the unique transformation given by $x^{\prime}=a x+b, a>0$, which transforms $A_{n k}$ onto $A_{n, k+1}$ for $k=1, \cdots, 2 n-1$, and $A_{n k}$ onto $A_{n, 1}$ for $k=2 n+2 . T$ is clearly a $1: 1$ point transformation of $S$ onto itself. Moreover it is easily seen that $T$ is measurable and pointwise periodic with the period of $2 n+2$ for the points of $c_{n}$. T also satisfies the following two conditions:

(1) $m\left(T^{-1} A\right) \leqq 2 m(A)$ for every measurable set $A$ in $S$.

(2) The set of ratios

$$
R_{A}^{n}=\frac{1}{2 n+2} \cdot \sum_{i=0}^{2 n-1} \frac{m\left(T^{-i} A\right)}{m(A)}
$$

where $n=1,2, \cdots$ and $A$ varies over all measurable sets $A \subseteq S$ is not a bounded set of numbers. (1) follows from the fact that $T^{-1}$ is de- 
termined by a linear transformation with a stretching factor of at most 2. (In fact, it is either $1 / 2,1$, or 2 .)

To prove (2) consider the sequence of sets $A_{n, n+1}$. Since $\bigcup_{i=0}^{2 n-1} m\left(T^{-i} A_{n, n+1}\right)=c_{n}$ we have that

$$
\frac{1}{2 n+2} \cdot \sum_{i=0}^{2 n-1} m\left(T^{-i} A_{n, n+1}\right)=\frac{1}{2 n+2} \cdot \frac{1}{2^{n}} \text {. }
$$

On the other hand $m\left(A_{n, n+1}\right)=\left(1 / 2^{n+1}\right) \cdot\left(1 / 2^{n}\right)$ and hence

$$
R_{A_{n, n+1}}^{n}=\frac{2^{n+1}}{2 n+2}
$$

which is an unbounded sequence.

We can now show that the above example satisfies the required conditions specified in the introduction.

(a) The individual ergodic theorem holds. In fact let $f(y)$ be any real-valued function defined on $S$, then since $T$ is pointwise periodic the sequence $\left\{F_{h}(y)\right\}$ converges to a finite limit for every $y$, that is, the individual ergodic theorem holds for every real-valued function defined on $S$. A fortiori it holds (with respect to $m$ ).

(b) $L_{1}(m)$ is transformed into itself by $T$ : In fact, it can be easily seen that $\bar{m}(A)=m\left(T^{-1} A\right)$ is a completely additive non-negative set function (that is, a measure) defined on the measurable sets of $S$. It can also be shown by considering approximating sums to the integrals that if $f \in L_{1}(m)$ then

$$
\int_{S}|f(T y)| d m=\int_{S}|f(y)| d \bar{m}
$$

By (1), $\bar{m}(A) \leqq 2 m(A)$ for every measurable set $A$ and hence

$$
\int_{S}|f(y)| d \bar{m} \leqq 2 \int_{S}|f(y)| d m<\infty
$$

from which follows that $f(T y) \in L_{1}(m)$, that is, $L_{1}(m)$ is transformed into itself by $T$.

(c) The mean ergodic theorem does not hold in $L_{1}(m)$. To prove this statement we use the following result due to Miller and Dunford $[3$, p. 539]: Suppose that the mean ergodic theorem did hold in $L_{1}(m)$; then there would exist a positive constant $c$ independent of $A$ and $h$ such that

$$
\frac{1}{h} \cdot \sum_{i=0}^{h-1} m\left(T^{-i} A\right)<c \cdot m(A)
$$


for all measurable sets $A$ and $h=1,2, \cdots$. But (i) is in contradiction with (2) above. Hence the mean ergodic theorem does not hold in $L_{1}(m)$.

It is possible to prove the last statement also directly by exhibiting functions in $L_{1}(m)$ for which the mean ergodic theorem does not hold in $L_{1}(m)$. In fact let $\bar{f}(y)$ be defined as follows: $\bar{f}(y)=2^{n}$ on $A_{n, n+1}, n=1,2, \cdots, \bar{f}(y)=0$ everywhere else on $S$, then $\bar{f}(y) \in L_{1}(m)$ but $\left\{\bar{F}_{h}(y)\right\}$ is not convergent in $L_{p}(m)$, for if it were then the limit function $\bar{f}^{*}(y)$ of $\left\{\bar{F}_{h}(y)\right\}$ would have to belong to $L_{1}(m)$. But $\bar{f}^{*}(y)$ is seen to be equal to $(1 / 2 n+2) \cdot 2^{n}$ for $y \in c_{n}$. We have

$$
\int_{S}\left|\bar{f}^{*}(y)\right| d m=\sum_{n=1}^{\infty}(1 / 2 n+2)
$$

which is a divergent series, that is, $\bar{f}^{*} \notin L_{1}(m)$ and hence $\left\{F_{h}(y)\right\}$ is not convergent in $L_{1}(m)$.

The example for $p \geqq 1$. Let $p$ be a fixed integer $\geqq 1$. Let $S$ be the same sequence of circumferences $c_{1}, c_{2}, \cdots$ as before. We divide each $c_{n}$ into $2\left(n \cdot 2^{p}-n+1\right)$ arcs, the end points being $x=0, x=1 / 2$ and $x=r / 2^{k p+1}, k=1,2, \cdots, n, r=1,2, \cdots, 2^{p}-1$. Again we define $T$ by the transformation given by $x^{\prime}=a x+b, a>0$, which transforms each arc into the next adjacent one.

$T$ is again seen to be a 1-1 measurable pointwise periodic transformation of $S$ onto itself with the period $2\left(n \cdot 2^{p}-n+1\right)$ for the points of $c_{n}$. As before it follows that (a) the individual ergodic theorem holds. (b) $L_{p}(m)$ is transformed into itself since there is a bound (the bound being $2^{p}$ ) on the stretching factor of $T^{-1}$. (c) The mean ergodic theorem does not hold in $L_{p}(m)$. To prove this last statement we use the following generalization of Miller and Dunford's result stated above: Let $t$ be any real number $\geqq 1$, then if the mean ergodic theorem holds in $L_{t}(m)$ there exists a constant $C$ independent of $A$ and $h$ such that

$$
\left[\frac{1}{h} \cdot \sum_{i=0}^{h-1} m\left(T^{-i} A\right)\right]^{t}<C \cdot m(A) .
$$

The proof is almost the same as for the special case $t=1$. If, however, we consider the sequence of sets $A_{n q}$, where $q=n\left(2^{p}-1\right)+1$ and where the enumeration of the $\operatorname{arcs}$ on each $c_{n}$ is analogous to that used in the case $p=1$, we can easily see that the sequence of ratios

$$
R_{n}=\left[\frac{1}{2 q} \sum_{i=0}^{2 q-1} m\left(T^{-i} A_{n q}\right)\right]^{p} / m\left(A_{n q}\right)
$$


is an unbounded sequence. This is in contradiction with (ii) for $t=p$. Hence the mean ergodic theorem does not hold in $L_{p}(m)$. Again the statement made in (c) may be proved directly by exhibiting functions in $L_{p}(m)$ for which the mean ergodic theorem does not hold in $L_{p}(m)$.

Let $p_{1}$ be any fixed number not less than 1 . Let $p$ be the first integer not less than $p_{1}$. Then the example constructed above for $p$ is also a valid example for $p_{1}$, for the individual ergodic theorem clearly holds and $L_{p_{1}}(m)$ is transformed into itself for the same reasons as before, while it follows from the fact that $m(A) \leqq 1$ for every measurable set $A$ and the fact that $p \geqq p_{1}$ that the same sequence of sets which violates (ii) for the case $t=p$ also violates (ii) for $t=p_{1}$. Hence the mean ergodic theorem does not hold in $L_{p_{1}}(m)$.

\section{BIBLIOGRAPHY}

1. G. D. Birkhoff, Proof of the ergodic theorem, Proc. Nat. Acad. Sci. U.S.A. vol. 17 (1931) pp. 356-660.

2. Yael Naim Dowker, Invariant measure and the ergodic theorems, Duke Math. J. vol. 14 (1947) pp. 1051-1061.

3. N. Dunford and D. S. Miller, On the ergodic theorem, Trans. Amer. Math. Soc. vol. 60 (1946) pp. 538-549.

4. J. von Neumann, Proof of the quasi-ergodic hypothesis, Proc. Nat. Acad. Sci. U.S.A. vol. 18 (1932) pp. 70-82.

RADCLIFFE CoLlege 